\title{
Assessing habitat connectivity for ground-dwelling animals in an urban environment
}

\author{
S. Braaker,,${ }^{1,2,3,6}$ M. Moretti, ${ }^{1,2}$ R. Boesch,${ }^{4}$ J. Ghazoul,${ }^{3}$ M. K. Obrist, ${ }^{1}$ and F. Bontadina ${ }^{1,5}$ \\ ${ }^{1}$ WSL Swiss Federal Research Institute, Biodiversity and Conservation Biology, Zürcherstrasse 111, CH-8903 Birmensdorf, \\ Switzerland \\ ${ }^{2}$ WSL Swiss Federal Research Institute, Community Ecology, Bellinzona, Switzerland \\ ${ }^{3}$ Department of Environmental Systems Science, ETH Zurich, Switzerland \\ ${ }^{4}$ WSL Swiss Federal Research Institute, Landscape Dynamics, Birmensdorf, Switzerland \\ ${ }^{5}$ SWILD, Urban Ecology and Wildlife Research, Zurich, Switzerland
}

\begin{abstract}
To ensure viable species populations in fragmented landscapes, individuals must be able to move between suitable habitat patches. Despite the increased interest in biodiversity assessment in urban environments, the ecological relevance of habitat connectivity in highly fragmented landscapes remains largely unknown. The first step to understanding the role of habitat connectivity in urban ecology is the challenging task of assessing connectivity in the complex patchwork of contrasting habitats that is found in cities.

We developed a data-based framework, minimizing the use of subjective assumptions, to assess habitat connectivity that consists of the following sequential steps: (1) identification of habitat preference based on empirical habitat-use data; (2) derivation of habitat resistance surfaces evaluating various transformation functions; (3) modeling of different connectivity maps with electrical circuit theory (Circuitscape), a method considering all possible pathways across the landscape simultaneously; and (4) identification of the best connectivity map with information-theoretic model selection. We applied this analytical framework to assess habitat connectivity for the European hedgehog Erinaceus europaeus, a model species for grounddwelling animals, in the city of Zurich, Switzerland, using GPS track points from 40 individuals.

The best model revealed spatially explicit connectivity "pinch points," as well as multiple habitat connections. Cross-validation indicated the general validity of the selected connectivity model. The results show that both habitat connectivity and habitat quality affect the movement of urban hedgehogs (relative importance of the two variables was $19.2 \%$ and $80.8 \%$, respectively), and are thus both relevant for predicting urban animal movements.

Our study demonstrates that even in the complex habitat patchwork of cities, habitat connectivity plays a major role for ground-dwelling animal movement. Data-based habitat connectivity maps can thus serve as an important tool for city planners to identify habitat corridors and plan appropriate management and conservation measures for urban animals. The analytical framework we describe to model such connectivity maps is generally applicable to different types of habitat-use data and can be adapted to the movement scale of the focal species. It also allows evaluation of the impact of future landscape changes or management scenarios on habitat connectivity in urban landscapes.
\end{abstract}

Key words: circuit theory; Circuitscape; city; connectivity; Erinaceus europaeus; fragmentation; generalized estimation equations (GEE); GPS tracking; habitat selection; hedgehog; landscape resistance; urban.

\section{INTRODUCTION}

Population persistence is facilitated by the degree to which organisms can move between resource patches (Taylor et al. 1993). Habitat connectivity, defined as the connectedness of habitat patches for a given species (Fischer and Lindenmayer 2007), assures access to food, refuge from predators, successful reproduction, and in

Manuscript received 13 June 2013; revised 1 November 2013; accepted 29 January 2014. Corresponding Editor: T. G. O'Brien.

${ }^{6}$ E-mail: sbraaker@gmx.net the longer term, dispersal and the maintenance of genetic diversity (Taylor et al. 2006). To apply efficient conservation measures for species populations in fragmented landscapes it is thus essential to identify important habitat connectivity pathways and barriers.

A critical step in every habitat connectivity analysis is the appropriate parameterization of the model, which means the building of the resistance surface. Resistance surfaces are most often parameterized based on expert opinion or literature review, but this approach has been criticized for its subjectivity (Spear et al. 2010, Sawyer et al. 2011). Attempts to avoid such subjective parameterization include basing movement resistance values on 


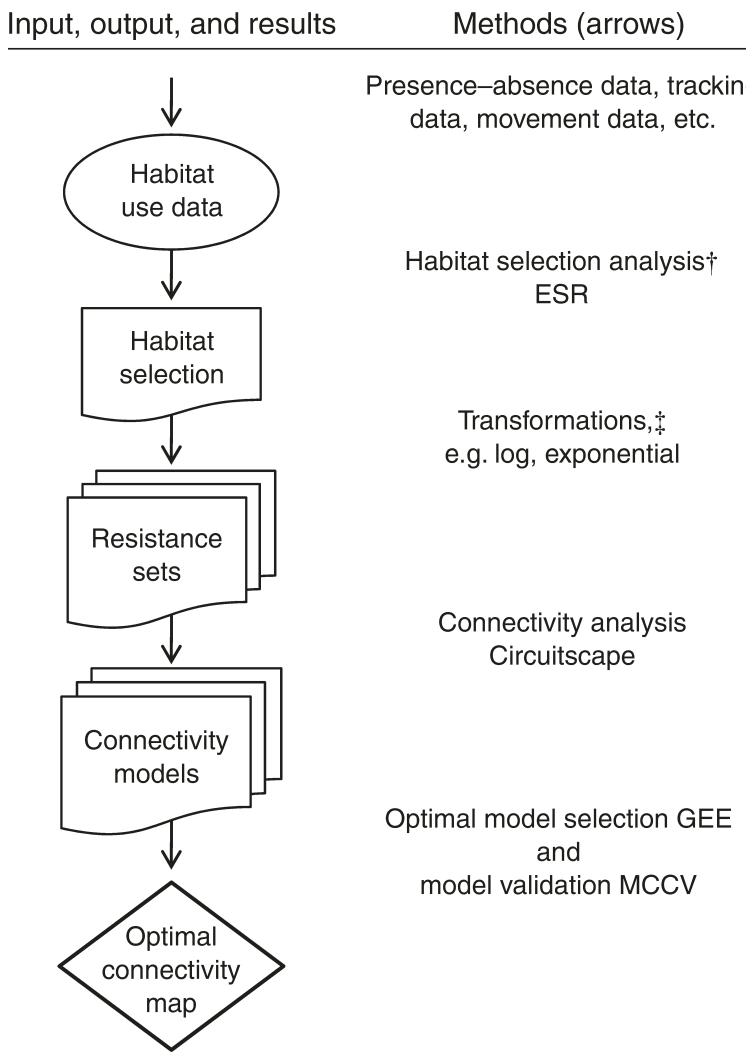

FIG. 1. General analysis framework to assess habitat connectivity applied to urban European hedgehogs (Erinaceus europaeus) in this study. The left column lists input and output data and results, and the right column shows the corresponding methods used (arrows). ESR stands for eigenanalysis of selection ratios, GEE for generalized estimation equations, and MCCV for Monte Carlo cross-validation (see Materials and methods section). Symbols in the methods column indicate where assumptions have to be checked before the framework is applied: The dagger $(\dagger)$ represents the assumption that habitatuse data reflects habitat used for movement; and the double dagger ( $\ddagger)$, the assumption that habitat selection is inversely related to movement resistance (see Discussion for details and possible alternatives if assumptions do not hold true).

occurrence data, movement paths, or experimental movement behavior data (Beier et al. 2008, Spear et al. 2010). The direction and functional form (e.g., positive, negative, linear, and logarithmic) of the relationship between resistance to movement and habitat quality is also not clear. To handle this uncertainty, a careful optimization approach can be used to select the most suitable resistance set from several alternatives (Spear et al. 2010, Trainor et al. 2013). In landscapes with a high variety of different habitats, and therefore resistance values, the outcome of the connectivity analysis might change drastically depending on the correlation function (Beier et al. 2008).

Frequently used connectivity metrics based on spatial patterns, such as the number of suitable patches within a radius, patch area, or distance to its nearest suitable neighbor (for overviews see Calabrese and Fagan 2004,
Saura and Pascual-Hortal 2007), all assume a binary classification of the habitat into suitable and hostile parts. As an alternative, least cost path (LC) methods model only a single best connection between a usually small number of suitable patches (e.g., Urban and Keitt 2001, Adriaensen et al. 2003). Neither assumption is likely to apply in a strongly fragmented area with many different land cover types. However, a new method based on electrical circuit theory (Circuitscape; McRae et al. 2008) is well adapted to assess connectivity for complex habitat patchworks. As in LC models, in Circuitscape, connectivity is also calculated based on a resistance surface in which contrasting resistance values reflect the level of suitability for an individual animal to move through a certain habitat (Sawyer et al. 2011). In contrast with LC, however, Circuitscape considers all possible pathways simultaneously to describe connectivity and permeability of the entire landscape. To minimize the subjective assumptions taken to make habitat connectivity analysis, we proposed a unique combination of four sequential steps in a data-based framework: (1) identification of habitat preference based on empirical habitat-use data, (2) derivation of habitat resistance surfaces evaluating various transformation functions, (3) modeling of different connectivity maps with electrical circuit theory (Circuitscape), and (4) identification of the best connectivity map with information-theoretic model selection (Fig. 1).

Habitat fragmentation is particularly pronounced in urban areas, where the landscape is generally characterized by ongoing disturbances and constant habitat transformations (Grimm et al. 2008). With the predicted rise of global human population from over $50 \%$ today to $69 \%$ in 2050 (United Nations 2012), it is likely that urban sprawl and also fragmentation increase accordingly. Despite the extreme conditions in urban landscapes, the wide variety of habitat types in cities often support a considerable number of animal and plant species (Sattler et al. 2010, Ramalho and Hobbs 2011). To maintain and actively promote biodiversity and to mitigate the fragmentation effects of increasing urbanization, it is essential to identify important connectivity pathways that can be integrated in urban planning and management strategies.

In this study, we developed a general, data-based analysis framework to assess habitat connectivity and apply this framework to an urban landscape using an extensive set of high-precision GPS tracking data on the European hedgehog Erinaceus europaeus (Linnaeus 1758 ) in the city of Zurich, Switzerland.

\section{Materials and Methods \\ Model species and study area}

European hedgehogs are nocturnal, solitary insectivores and have non-territorial, overlapping home ranges of 10 to 40 ha (Bontadina et al. 1994, Morris and Reeve 2008). Home ranges of males are usually larger, and, especially in the breeding season, females are more 
stationary, however sexes do not differ in their general pattern of habitat use (Rondinini and Doncaster 2002, Riber 2006). The individual home range can shift over several months and years, but hedgehogs do not show a clear dispersal phase in their life history (Reeve 1994, Doncaster et al. 2001). Hedgehogs are opportunistic, feeding mainly on a wide range of invertebrates, and spend most of their nightly activity foraging (60-80\%), nevertheless covering large distances (1000-1500 m; Zingg 1994, Riber 2006). During the day, they use various structures such as heaps of leaves or wood, places below buildings, hedges, and forested areas with dense understory as resting sites, which they often change daily. In urban settlements, hedgehog densities tend to be higher than in rural areas, which has been related to agricultural intensification and reduced predation by badgers in urban areas (Bontadina et al. 1994, Young et al. 2006).

We conducted the study in Zurich $\left(47^{\circ} 22^{\prime} 0^{\prime \prime} \mathrm{N}\right.$, $\left.8^{\circ} 33^{\prime} 0^{\prime \prime} \mathrm{E}\right)$, the largest city of Switzerland, located next to a lake on the Swiss central plateau. The greater Zurich urban area is $91.9 \mathrm{~km}^{2}$ and has more than one million inhabitants. It contains densely built-up residential and industrial areas, but also a large proportion of green spaces $(37.5 \%)$, ranging from forests to parks, gardens, and agricultural land (Hennig and Ghazoul 2011).

\section{Habitat map}

A detailed GIS habitat map of Zurich with a spatial precision of $<10 \mathrm{~m}$ (Biotoptypenkartierung der Stadt Zurich 2010) was combined with exact GIS layers of buildings and streets from the Swiss Federal Office of Topography (Vector25; Swisstopo 2011). The resulting nonoverlapping 197 land cover classes were merged into 20 habitat classes relevant for hedgehogs (see Appendix A: Table A1). The original vector map was transformed into a raster map with a cell size of $2 \times 2 \mathrm{~m}$. All analyses involving GIS layers were performed in ArcMap 9.3.1 (ESRI 2009). For calculations and statistical analyses, R version 2.13.0 (R Development Core Team 2011) was used, unless otherwise stated.

\section{GPS tracking of hedgehogs}

Twelve locations across the city were chosen as starting points to search for hedgehogs. Two times six locations were chosen at random in areas of high and low hedgehog density, respectively (Bontadina et al. 1994). The study focused on male hedgehogs because they have more extensive home ranges and are thus more suitable for investigating the effects of landscape connectivity. Between May and September 2009, hedgehogs were captured and fitted with a custom-built GPS transmitter fixed with epoxy resins onto the shortened spines of the lower back of the hedgehog. After the tracking period of several nights, the transmitter was removed. The GPS transmitter consisted of a GPS logger (ML-7 unit; SANAV San Jose Technology,
Taiwan), a conventional VHF radio transmitter (Titley Electronics, Australia, or Wagener Telemetrieanlagen, Germany), and an extended LiPo battery. The total mass of the transmitter was 24-34 g, which amounted to $1.9-4.9 \%$ of the mass of the hedgehogs (recommended limit is 5\%; White and Garrott 1990). The GPS loggers were programmed to store the recorded positions of hedgehogs every $10 \mathrm{~s}$. An extensive field test revealed location errors (circular error probability, $N=6480$ ) of $50 \%$ of locations $<5.4 \mathrm{~m}$, and $90 \%<10.0 \mathrm{~m}$ in open space (park) and $50 \%$ of locations $<15.3 \mathrm{~m}$, and $90 \%$ $<25.0 \mathrm{~m}$ in a covered place (backyard). Precision of recorded position is expected to be higher for moving animals due to a movement optimizing algorithm integrated in the logger by the manufacturer. We relocated animals through the radio signals to retrieve GPS data by exchanging the data logger on a nightly basis. For the analyses, we used only the locations recorded between sundown and daybreak (nocturnal activity) and removed imprecise GPS locations by deleting all locations implying an unrealistic speed higher than $1 \mathrm{~m} / \mathrm{s}$ (Bontadina 1991) and those recorded erroneously in an inaccessible habitat such as buildings.

\section{Habitat selection analysis}

Because hedgehogs spend most of the night foraging while constantly moving, the habitat used for moving is likely to be similar to the habitat used for foraging. To identify preferences for defined habitat classes, we used the eigenanalysis of selection ratios (ESR), a multivariate analysis based on the ratio of used-to-available habitats (R package 'adehabitat'; Calenge 2006, Calenge and Dufour 2006). In contrast to the more common compositional analysis (Aebischer et al. 1993), ESR weights individuals by the number of recorded GPS locations. With the ESR method, a ranking as well as a quantitative measure of relative habitat selection can be derived. Because we were interested in hedgehogs' finescale movement, we defined for each individual hedgehog the "available" habitat as the minimum convex polygon (MCP) around all its GPS locations, with a 25$\mathrm{m}$ buffer (according to design III of Manly et al. 2002, Beyer et al. 2010). The size of the buffer was conservatively chosen according to the estimated $90 \%$ circular error probability in covered places, which was $25 \mathrm{~m}$. Six habitat classes (highway, forest, marsh, small water, large water, and building) were excluded from the ESR because they were either not used by hedgehogs or not present in MCPs. An average habitat selection axis was derived from the sum of vectors of individuals on the first two selection axes. The relative position of the habitats projected on the average habitat selection axis gives a quantitative measure of habitat selection (for details, see Fig. 2 and Appendix B).

\section{Habitat connectivity analysis}

Circuitscape couples graph theory with electrical circuit theory and measures habitat connectivity by 

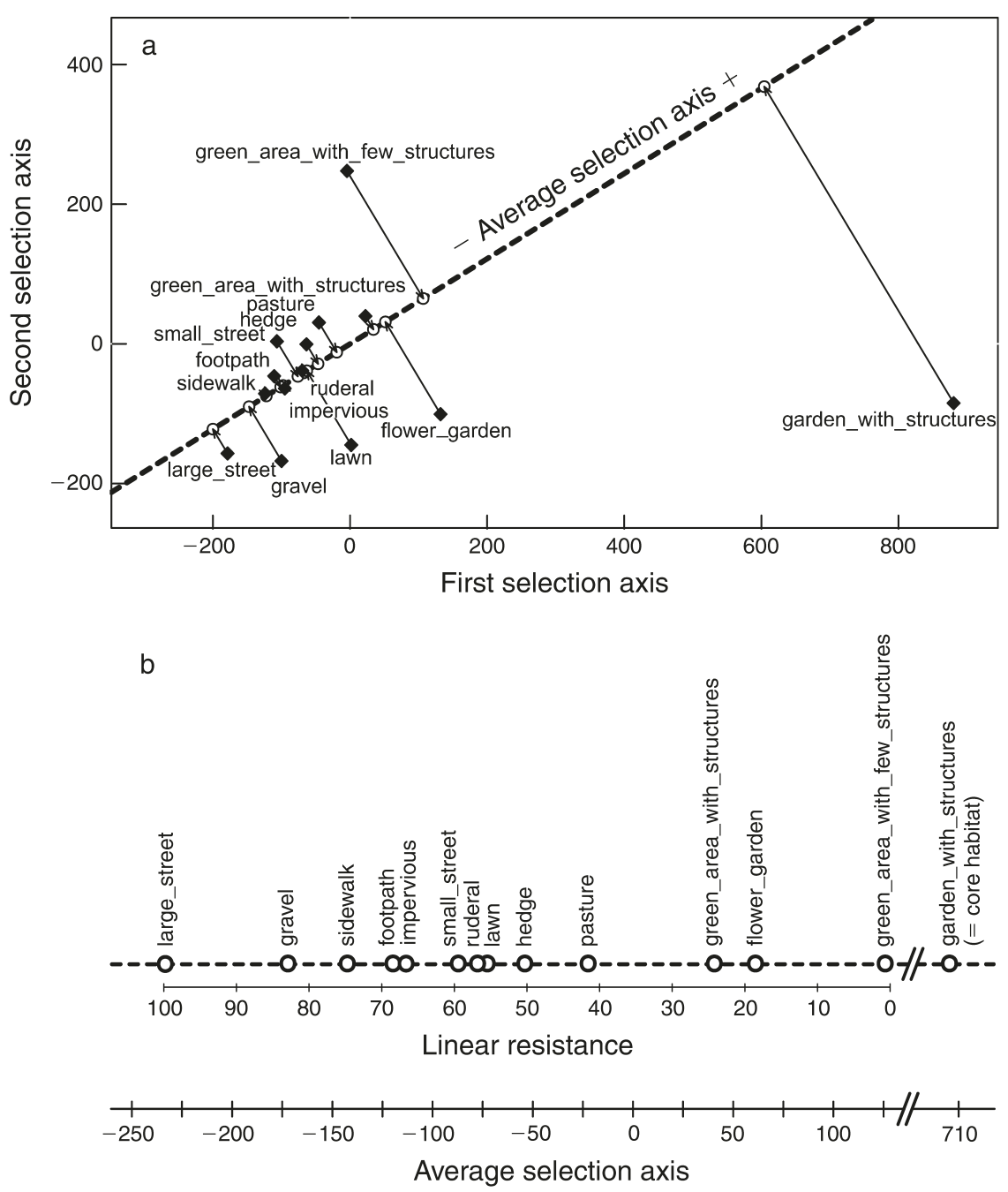

FIG. 2. (a) Eigenanalysis of selection ratios of 40 hedgehogs in 14 habitats. The position of the habitats on the first two selection axes is shown as black diamonds. The dashed line represents the average selection axis. Habitats are projected orthogonally on the average selection axis (arrows and open circles). (b) Ranking and relative strength of the selection of the habitats along the average selection axis. The most selected habitat, "garden_with_structures," is designated as the core habitat (node) for all Circuitscape analyses. The linear habitat resistance axis is the linear inverse of the average selection axis rescaled from 1 to 100 .

calculating the cumulative current that flows through each cell of a resistance map between a given set of nodes, which are set pairwise as sources of current and ground (McRae 2006, McRae et al. 2008). In the analyzed landscape, current flow is calculated between every possible pair of nodes (all other nodes are meanwhile set to minimal resistance value one), and the resulting current maps are added up to a single cumulative current map. Measures of electrical circuits (current, voltage, resistance) have been shown to have a precise relationship with random walk theory, which facilitates their ecological interpretation (McRae et al. 2008). In ecological terms, nodes are suitable core habitats and the resistance value of a cell represents the likelihood of a random walker to choose to move through this cell (McRae et al. 2008). Current flowing through a resistance cell can be interpreted as the expected net movement probability for a random walker to move through this cell and is determined by the configuration and resistor values of the resistance surface in the circuit. The more alternative pathways that exist to move between core habitats, the broader is the random walker distribution, and therefore, the lower the current flowing along any single path. The fewer alternative pathways exist, the higher the current flowing through the existing paths. High current areas thus highlight connectivity pinch points in the landscape. Because Circuitscape is based on electrical circuit theory, it has the advantage that both minimum movement cost and alternative pathways are taken into account to predict movements (McRae et al. 2008).

For our analysis, we defined contiguous cells of a hedgehog's most selected habitat as core habitat patches. Based on a translocation experiment and field 


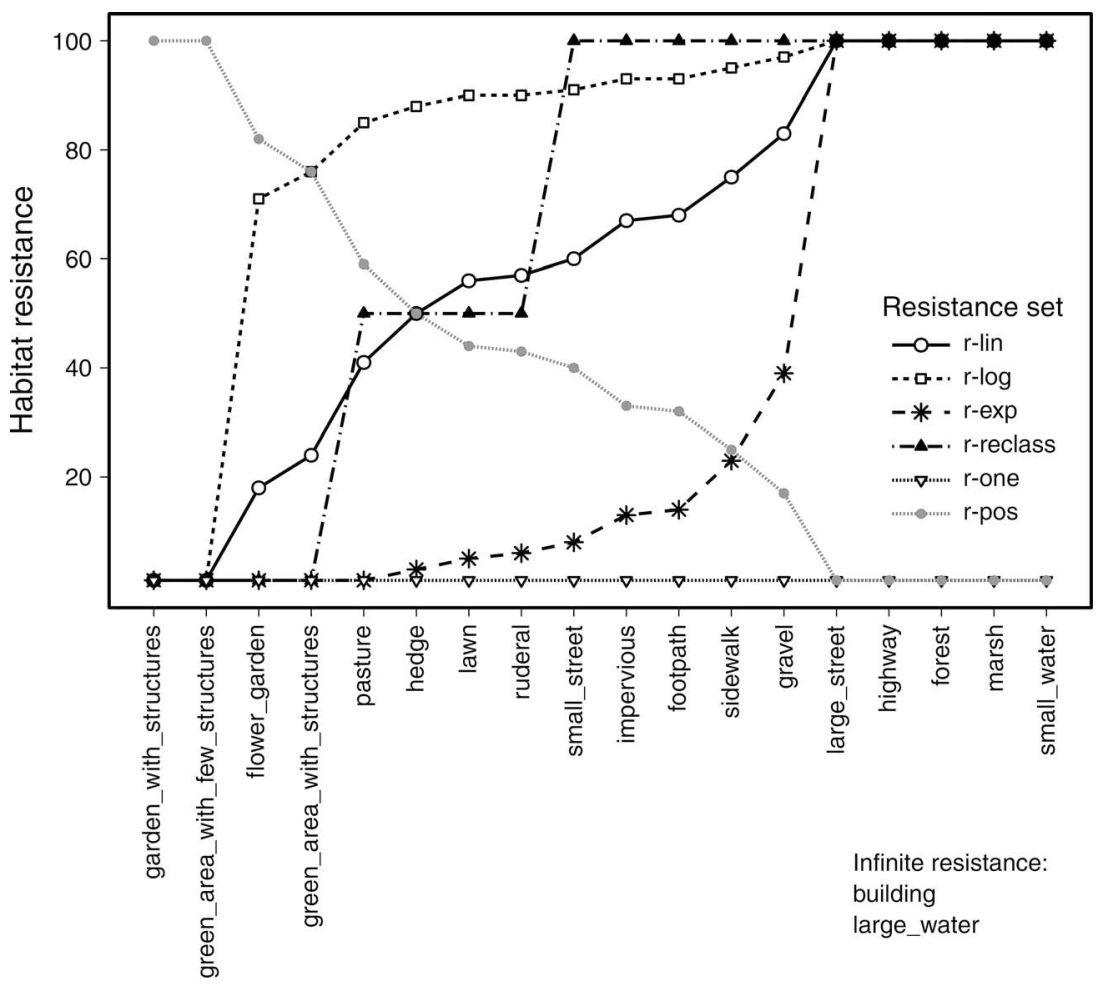

Habitats

FIG. 3. Habitat resistance values assigned to habitats in six alternative resistance sets, rescaled from 1 to 100. Different symbols represent values of alternative resistance sets. Symbols are only joined by lines for clarity. Resistance values are deduced from habitat selection analysis (see Fig. 2). Abbreviation of resistance sets are: r-lin, linear inverse of the rescaled habitat selection values; r-log, natural logarithm of r-lin; r-exp is r-lin raised to the power of five; r-reclass, reclassification of r-lin in three classes; r-one, all habitats set to resistance 1; and r-pos, rescaled habitat selection values (positive relationship with habitat selection). The habitats excluded from habitat selection analysis (highway, forest, marsh, small water) were set to the maximum resistance value 100 (except in r-one and r-pos).

observations that demonstrated significant faster movement of hedgehogs in unfavorable habitat than in favorable habitat (Bontadina 1991, Doncaster et al. 2001), we expected habitat resistance to be inversely, but not necessarily linearly, correlated to habitat selection (Beier et al. 2008, Spear et al. 2010). Higher resistance thus signifies a higher reluctance of an individual to choose to move through and spend time in a habitat type and consequently a lower selection of this habitat. Reasons could be elevated disturbance level or high mortality risk. This higher reluctance of an individual is not the consequence of a higher physical difficulty to move, which would result in slower movement, more time spent in the area, and greater habitat selection. To corroborate our assumption of an inverse selectionresistance relationship, we first built two resistance sets "r-lin" (r, resistance; lin, linear) with values based on the linear inverse of habitat selection values, and "r-pos" derived from linear positive habitat selection values ( $r$, resistance; pos, positive relationship; Fig. 2). We then compared the Circuitscape current maps "c-lin" and "cpos" (c, current), which were calculated based on these two resistance sets. For the main analyses, we focused on an inverse relationship and derived three additional resistance sets with values between 1 and 100 based on correlation functions representative of the possible functions linking habitat selection and habitat resistance (Fig. 3). Additionally, we built one neutral set where habitat type was assumed to have no effect. The final five resistance sets used for modeling were: (1) "r-lin," linear resistance described above; (2) "r-log," r-lin transformed with the natural logarithm; (3) "r-exp," rlin raised to the power of five; (4) "r-reclass," the reclassification of habitats along r-lin in three classes; and (5) "r-one," the control set with all habitats set to resistance one. We hypothesized that habitats excluded from the habitat selection analysis (highway, forest, marsh, small water) represent areas that are difficult, but not impossible, for urban hedgehogs to cross, and thus, these habitats were set to the maximum resistance value 100 (except in r-one). Buildings and large water bodies, such as the Lake Zurich, were assumed to be absolute barriers to movement and given infinite resistance (no current flows through) in all five resistance sets.

Artificial map boundaries are known to have a strong effect on current flow through the landscape (Koen et al. 
2010). Therefore, a $2-\mathrm{km}$ buffer of randomized habitat with the same habitat composition as in the map of Zurich was added around the city (where the detailed habitat maps were not available; Koen et al. 2010). The maximum dimension of the solvable circuit surface with a $2 \times 2 \mathrm{~m}$ cell size, using focal points as nodes and cell connections limited to four neighbors (the fastest options) was computationally limited to a square of 9 $\mathrm{km}^{2}$ (calculation time $\sim 16 \mathrm{~h}$ ). We thus selected 14 resistance surfaces of $9 \mathrm{~km}^{2}$ in a way that the MCP of each individual was covered by one of them. To avoid boundary effects of the Circuitscape method, an outer $500-\mathrm{m}$ buffer was removed from the calculated $9-\mathrm{km}^{2}$ Circuitscape maps, resulting in $4-\mathrm{km}^{2}$ maps for further analyses.

\section{Best model selection}

We formulated competing models for predicting hedgehog movement points based on connectivity (current flow), habitat quality (habitat selection), and connectivity plus habitat quality. Because a random walker is more likely to move through high current cells (McRae et al. 2008), we hypothesized the hedgehogs' GPS track locations also more likely to be located in high current cells than in randomly selected locations. Based on this assumption, we formulated logistic regression models (Manly et al. 2002) with GPS track locations and random locations as binary response variable and current values of the different connectivity models as single explanatory variables (connectivity models). We assumed a model to be valid when the coefficient has a positive sign. We were also interested in knowing whether both habitat connectivity and habitat quality improved the performance of the model to assess animal movement between core habitat patches. Assuming that ESR habitat selection is a measure of habitat quality for the hedgehog, we built one logistic regression model with habitat selection as the single explanatory variable (habitat quality model) and five models with habitat selection and current values of each connectivity model as explanatory variables (connectivity plus habitat quality models). We randomly selected the same number of locations as GPS locations in each MCP (Hawth's tool for ArcGIS; available online). ${ }^{7}$ Current values and habitat selection values were assigned to all GPS and random locations, but we excluded those on core habitat patches (likely to be the habitat most intensively used for foraging) because our main focus was on inter-patch connectivity. All values were $z$-normalized to facilitate a comparison of regression coefficients.

To account for the inherent autocorrelation between GPS locations, we used generalized estimating equations (GEEs; R packages "yags" and "geepack"; Liang and Zeger 1986, Carey 2004, Halekoh et al. 2006). These

${ }^{7}$ http://www.spatialecology.com/htools extend generalized linear models by including an additional variance component for correlation structure within data clusters. GEEs are highly recommended if the focus is on a population's averaged response (Fieberg et al. 2009, Koper and Manseau 2009). We designated individual animals as clusters and assumed the correlation structure to be independent (Craiu et al. 2008, Fieberg et al. 2010). The quasi-likelihood under the independence model information criterion (QIC; Pan 2001), a generalization of the Akaike information criterion (AIC), is suitable for model selection of GEEs (Hardin and Hilbe 2003). As for AIC, all models are compared to the model with the lowest QIC score to compute delta QIC. Models with delta QIC values larger than 10 have essentially no support (Burnham and Anderson 2002). Due to computational limitations, we could use only every second recorded GPS point for the calculations of GEEs.

\section{Model validation}

To verify that the best connectivity and connectivity plus habitat quality models were not only the best models of some poor candidate models (Spear et al. 2010), but were also efficient in predicting connectivity and animal movement of urban hedgehogs, we applied a Monte Carlo cross-validation (Xu and Liang 2001). Given the long computation time (four minutes per model), we repeated Monte Carlo cross-validation and the subsequent ranking test only 100 times. We randomly split the individuals into two sets (training and validation set), using half of the data as the training set. This reduces the chance of overfitting and makes the model generally a better predictor of independent data (Xu and Liang 2001). Spearman's rank correlation with algorithm AS 98 approximation (R package "pspearman"; Savicky 2009) was performed on adjusted frequencies across 10 equal-area bins (similar to Boyce et al. 2002). High rho values indicate an increasing number of hedgehog GPS points assigned to upper bins (bins with higher current values). Additionally, we compared the proportions of overlap of high current areas (current $\geq$ model mean + one standard deviation) in the 14 squares for all model pairs.

\section{Results}

Hedgehog tracking and habitat selection analysis

Forty male hedgehogs were tracked with GPS loggers between one and six nights for a total of 727 hours of activity (Appendix C: Table C1). After removing erroneous locations from the GPS tracks, between 307 and 44881 locations per animal and a total of 499641 locations were available for the analyses (Appendix C: Table $\mathrm{C} 1$ ). The average nightly activity range, calculated as the mean area of individual MCPs, was 17.32 ha (SE $=3.04$ ), covering a total urban area of 517 ha. The composition of the available habitat areas (buffered MCPs of all individuals) revealed that we tracked 
a

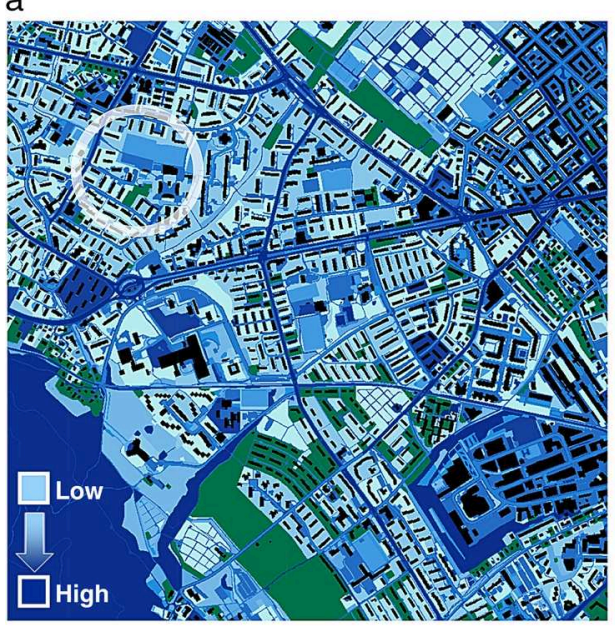

C
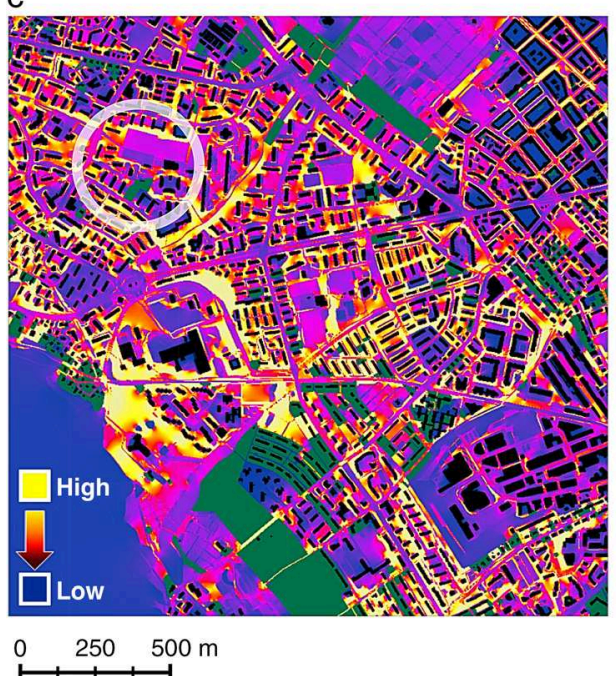

b

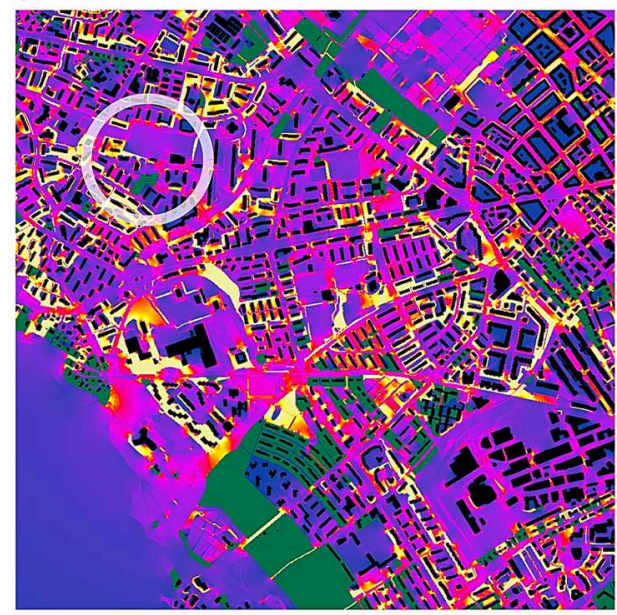

d

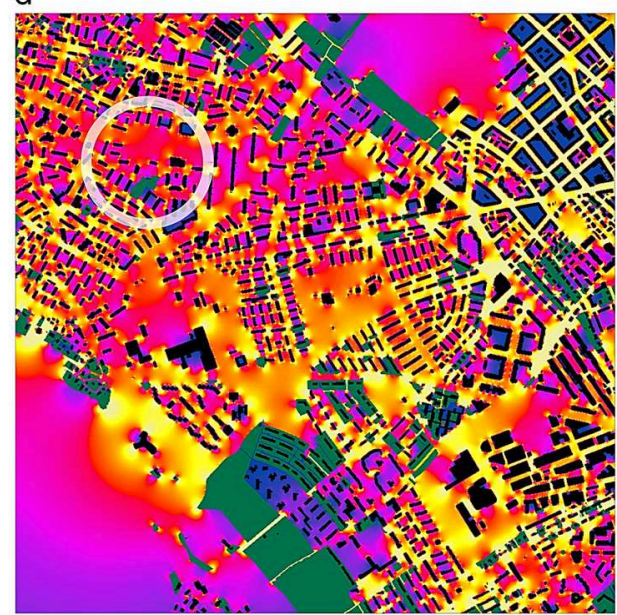

FIg. 4. Example of one of the 14 raster surfaces $\left(4-\mathrm{km}^{2}\right.$ squares, cell size $\left.2 \times 2 \mathrm{~m}\right)$ used to calculate hedgehog habitat connectivity maps. (a) Resistance map of the linear resistance set r-lin. Light blue indicates low resistance, dark blue high resistance, and black infinite resistance. Core habitat patches are represented in green. (b-d) Standardized cumulative current maps modeled with Circuitscape. Current modeled with (b) linear resistance (c-lin); (c) exponential resistance (c-exp); and (d) resistance one (cone). Yellow represents high current flow (connectivity), and dark blue represents low current flow. Bright yellow areas highlight pinch points and indicate essential connectivity areas. Core habitat patches are colored in green and infinite resistance values in black. The circles mark the same area on all four maps to facilitate the comparison between maps.

hedgehogs in an urbanization gradient ranging from $10 \%$ to $72 \%$ of built-up and impervious area.

The first habitat selection axis of the ESR analysis explains $25.6 \%$ of the total variation in habitat selection, and the second $18.7 \%$ (Fig. 2a). Average habitat selection ranged from most avoided habitat "large streets" $(-150)$ to most selected habitat "garden with structures" (+710) (Fig. 2b). The latter was defined as the core habitat for the subsequent Circuitscape analyses. Resistance surfaces of one positive and five inverse resistance sets were obtained by transforming and rescaling the average habitat selection values (Figs. 3 and $4 a)$.

\section{Habitat connectivity and animal movement models}

The coefficient of the connectivity model c-lin was positive $(0.08)$, while the model c-pos revealed a strong negative coefficient $(-0.17)$, corroborating the assumption of an inverse relationship between habitat selection and resistance. Therefore, we focused on the five models based on resistance sets with an inverse relationship. Pairwise comparison of the high current areas in the five alternative connectivity maps (contrasting examples are shown in Fig. 4b-d) revealed relatively low proportional overlap of 0.11 to 0.78 (Appendix D: Table D1). This signifies that different resistance sets lead to different connectivity maps. Comparison between the competing 
TABLE 1. Description of 11 fitted generalized estimating equations (GEEs).

\begin{tabular}{lrrrrr}
\hline \hline \multicolumn{1}{c}{ GEE model } & QIC & Delta QIC & Variable & $\beta$ & SE \\
\hline a) Habitat connectivity models & & & & & \\
c-lin & 175655 & 340 & c-lin & 0.08 & 0.03 \\
c-log & 175725 & 409 & c-log & 0.06 & 0.03 \\
c-exp & $\mathbf{1 7 5 3 1 5}$ & $\mathbf{0}$ & c-exp & $\mathbf{0 . 1 3}$ & $\mathbf{0 . 0 3}$ \\
c-reclass & 175408 & 93 & c-reclass & 0.12 & 0.03 \\
c-one & 175841 & 525 & c-one & -0.01 & 0.03 \\
b) Connectivity plus habitat quality models & & & & \\
s-lin & 174188 & 21 & s-lin & 0.23 & 0.04 \\
c-lin + s-lin & 174220 & 53 & c-lin & -0.02 & 0.02 \\
& & & s-lin & 0.24 & 0.04 \\
c-log + s-lin & 174225 & 59 & c-log & -0.03 & 0.03 \\
& & & s-lin & 0.24 & 0.04 \\
c-exp + s-lin & $\mathbf{1 7 4 1 6 7}$ & $\mathbf{0}$ & c-exp & $\mathbf{0 . 0 5}$ & $\mathbf{0 . 0 3}$ \\
c-reclass + s-lin & & & s-lin & $\mathbf{0 . 2 1}$ & $\mathbf{0 . 0 4}$ \\
& 174205 & 39 & c-reclass & 0.03 & 0.03 \\
c-one + s-lin & & & s-lin & 0.22 & 0.04 \\
& 174207 & 40 & c-one & 0.02 & 0.03 \\
& & & s-lin & 0.24 & 0.03
\end{tabular}

Notes: Quasi-likelihood under the independence model information criterion (QIC) is a measure of model fit, where models with delta QIC $>10$ have essentially no support. Models with the smallest QIC are highlighted in boldface type. Also given are GEE fixed-effects estimates $(\beta)$ and empirical standard errors (SE) of the explanatory variables. Model names and variable names are composed of the type of value (c represents current, and s represents habitat selection) and the type of transformation performed on the underlying values (lin, linear; log, natural logarithm; exp, raised to power of five; reclass, three classes; and one, all values equal one).

connectivity, habitat quality, and connectivity plus habitat quality models demonstrated that connectivity was best modeled with the exponential connectivity model (c-exp, delta QIC of second best model = 93; Table 1a), which showed only $45 \%$ overlap of high current areas with the second best model c-reclass. The habitat of the hedgehog was best modeled with the connectivity plus habitat quality model "exponential current" and "linear habitat selection" (c-exp + s-lin, delta QIC of second best model = 21; Table 1b, Fig. 5). A comparison of the standardized coefficients of the model c-exp $+\mathrm{s}$-lin revealed that the relative importance of habitat connectivity in predicting animal movement was $19.2 \%$, compared to $80.8 \%$ contributed by habitat quality.

The evaluation of model performance by crossvalidation and subsequent Spearman's rank test showed a significant positive correlation coefficient rho for the connectivity model c-exp (rho $=0.75, \mathrm{SD}=0.2, P \leq$ 0.05 ), as well as for the connectivity plus habitat quality model c-exp + s-lin $($ rho $=0.64, \mathrm{SD}=0.18, P \leq 0.05)$.

\section{Discussion}

Our multistep framework is among the first to successfully identify spatially explicit connectivity pathways for animals in highly fragmented and heterogeneous environments. The modeled connectivity maps demonstrate that in cities, both connectivity and habitat quality play a substantial role in influencing the movements of ground-dwelling animals. Our results provide a new view of animal movements in complex habitat matrices, and the importance of connectivity in urban habitats indicates its relevance for ecological landscape planning.

\section{Challenges and opportunities to assess urban habitat connectivity}

The importance of habitat connectivity for animal movements in fragmented and heterogeneous landscapes such as urban areas has been little studied until now. In cities, land cover types are extremely diverse and independent patches are very small (usually a few square meters), what bears simultaneous opportunities and methodological challenges to study habitat connectivity. Diverse landscapes provide an opportunity to study the whole range of land cover types ranging from low to high movement resistance. But at the same time, the strong heterogeneity requires a high temporal and spatial resolution of movement data, and it necessitates analysis methods that consider the complex landscape configuration. Our innovative approach deals with these challenges by combining GPS tracking, which makes it possible to collect frequent location records with high spatial resolution, with precise land cover maps, advanced modeling techniques and topical statistical tools.

Circuitscape relies on resistance surfaces and considers multiple paths simultaneously, which makes it well adapted to modeling connectivity in a complex urban environment. Although Circuitscape has become popular, to date, only few other studies have used GPS technology to parameterize resistance input maps for Circuitscape (Poor et al. 2012, Shafer et al. 2012), but never in an urban environment. Our evaluation suggests that different translations of habitat selection into 
a

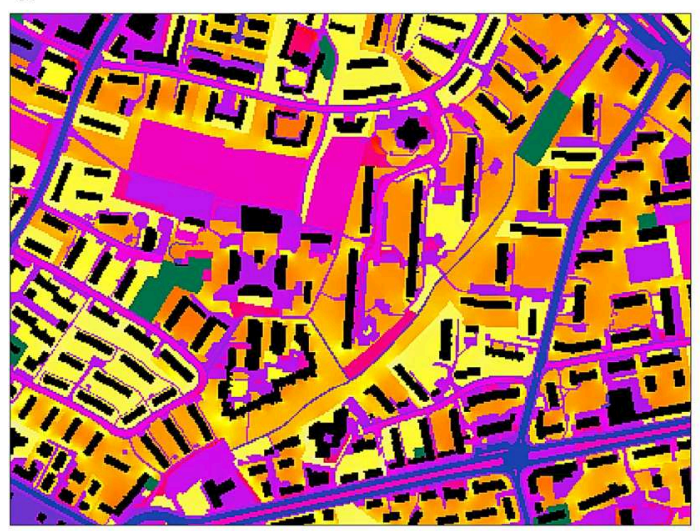

C

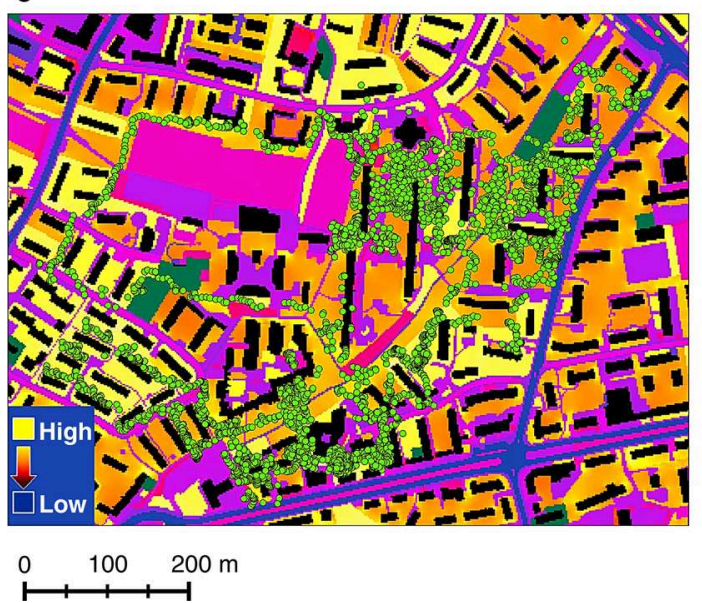

b

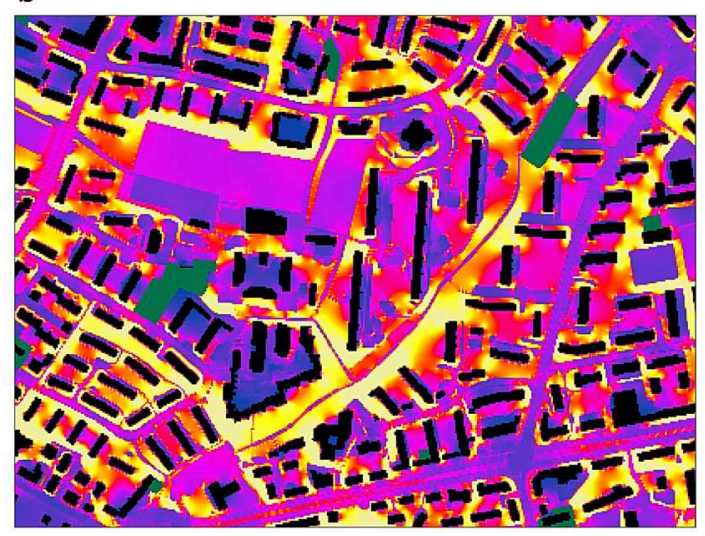

d

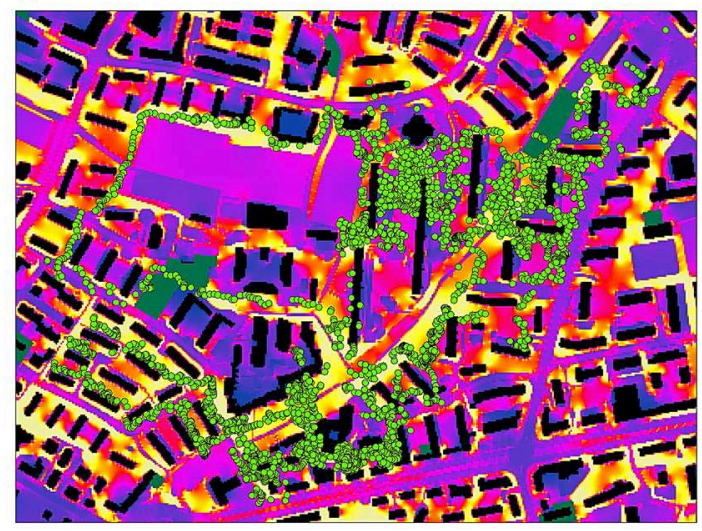

FIG. 5. For comparison, an example of the (a) best predicted connectivity plus habitat quality map (c-exp + s-lin) and (b) best predicted connectivity map (c-exp), calculated from GEE fixed-effects coefficients. High values (e.g., current) are colored yellow, low values are in blue, and habitats of infinite resistance are black. Source habitats are plotted in green. A GPS track of one hedgehog from five nights is represented with green dots on the same landscape in panels (c) and (d). Disparities between the maps illustrate the different information that the complementary predicted maps contain, i.e., habitat use vs. pinch point connections.

resistance values substantially influence the location of the modeled connectivity pathways in the Circuitscape maps. Given the only marginal overlap of these pathways between the alternative models, it is thus essential to test different possible translations and not only a simple linear correlation. Our analysis revealed an exponential correlation function between habitat selection and resistance. This is in agreement with Trainor et al. (2013), who tested a range of seven transformation functions and also found a much better performance of a nonlinear (resembling an exponential) transformation function. We might have obtained more accurate predictions by testing more translation functions using an iterative approach to find the model with lowest QIC value. The high computational effort to calculate detailed Circuitscape maps is actually limiting this approach.

Habitat quality is known to play a key role in habitat use (Manly et al. 2002), but the relative importance of connectivity for population persistence is disputed (Hodgson et al. 2009, Doerr et al. 2011). In our study, habitat connectivity was modeled as an interaction between the composition of the landscape, consisting of specific movement resistances, and their spatial configuration (McRae et al. 2008). Although we modeled habitat connectivity on the basis of movement within home ranges, given the biology of the study species (no territories, no distinct dispersal phase, and similar habitat use of sexes), it is likely that the connectivity pinch points for home range movement also facilitate the dispersal of individuals. To disentangle the contribution of habitat quality and habitat connectivity, we compared a movement model that incorporated both habitat quality and connectivity with a model that included habitat quality only, assuming that habitat selection is a measure of habitat quality. The information-theoretic model selection showed that the combined habitat quality and connectivity model performed better 
(Table 1b). Model coefficients of the best movement model demonstrated that for urban hedgehogs habitat connectivity is less important than habitat quality, but still explains almost $25 \%$ as habitat quality. In all tested models, habitat quality was consistently important. However, it is important to emphasize that the information provided by habitat connectivity models, which includes the aspect of spatial configuration of land cover types, complements that provided by habitat quality models. Further, the importance of habitat connectivity might even increase with further tuning of the translation function. Habitat quality indicates which areas are generally suitable for the species, whereas habitat connectivity highlights areas that are indispensable to ensure movement between suitable habitat patches (Fig. 5). Connectivity pinch points have major implications for management and conservation.

\section{Habitat selection and connectivity for urban hedgehogs}

The urban hedgehogs we studied showed a strong preference for gardens rich in structures, which, in addition to flower and vegetable beds, contain bushes, trees, and elements like heaps of branches or stones. Such a habitat harbors many invertebrates (Smith et al. 2006, Sattler et al. 2010). Gardens with structures, therefore, provide shelter and abundant food resources, not only for hedgehogs, but for numerous other urban dwellers as well (Goddard et al. 2010). Habitat selection showed that hedgehogs prefer urban green spaces with structures over lawn and pastures without structures, and impervious areas are least preferred. This is in accordance with previous studies describing habitat selection of hedgehogs (Bontadina 1991, Rondinini and Doncaster 2002, Dowding et al. 2010). The exponential correlation we found between habitat selection and resistance indicates, however, that all green spaces and impervious areas with little traffic present relatively low resistance to movement. This contrasts strongly with main streets, which acted as major movement barriers (see also Rondinini and Doncaster 2002).

Our results suggest that increasing the number of large gardens and parks, both rich in structure, can promote urban hedgehogs. This measure would certainly benefit not only hedgehogs, but also many other urban dwellers (Goddard et al. 2010). However, in historical cities, green areas tend to be frequent, but small and highly fragmented (Goddard et al. 2010). Restoring large, high-quality habitat patches, as suggested by Hodgson et al. (2009), is rarely possible due to lack of space. With these restrictions, connectivity maps provide valuable evidence-based information about priority areas for conservation and management measures to improve connectivity.

\section{Implications of connectivity maps}

The visualization of connectivity in spatially explicit maps uncovers a fundamental, but otherwise invisible, ecological factor of major relevance for animal movement. If connectivity pinch points are transformed into barriers, animals' movements between habitat patches, or even whole regions, will be inhibited. This may lead to increased fragmentation with severe consequences for population persistence (Rayfield et al. 2011). However, if multiple pathways connect habitat patches, the transformation of a single pathway will have less consequence on overall connectivity of the landscape. Multiple pathways are thus increasing the resilience of the habitat network and are desirable from a conservation-planning perspective (Urban et al. 2009, Laita et al. 2011). Connectivity maps of Circuitscape highlight the pathways crucial to maintain overall connectivity (McRae et al. 2008). Low current areas, however, can represent either high-resistance areas (barriers) or large swaths of low-resistance cells (large corridor), because both reduce current flow in a single cell. When interpreting connectivity maps, it is important to remember that their focus is on connectivity pinch points, i.e., narrow corridors leading to high current flow. The distinction between barriers and large corridors (both low current areas) is only possible with the corresponding resistance map.

\section{The general applicability of the analysis framework}

Habitat connectivity models are important to define management strategies and several studies proposed approaches to deal with the problematic use of subjective assumptions taken at different steps of the analysis. Some used radio-tracking or genetic data to select the best resistance values out of a limited set, which was based on expert knowledge (Cushman et al. 2006, Richard and Armstrong 2010). Some used resistance values based on expert knowledge, but then varied the resistance values according to defined model functions and applied Circuitscape modeling (Shirk et al. 2010). And others derived habitat suitability from radio-tracking data, and then tested various transformation functions to define the resistance surface and validated the final model with a separate set of data (Trainor et al. 2013), but applied least cost path modeling, which calculates only a single optimal path.

The multistep framework we developed in this study is unique in the way it combines different objective analysis methods. It is especially suitable for landscapes with a variety of different land cover types that all might have distinct effects on the investigated animal species. It can be applied to different types of habitat-use data (e.g., presence absence data, radio- or GPS-tracking data), depending on the species in focus and the data available (Calenge 2006, Horne et al. 2007, Kranstauber et al. 2012). The scale of the investigated landscape is adaptable to the movement range of the species studied and to the type of connectivity assessed (e.g., within home range movement, long-distance dispersal, or migration). Habitat connectivity is species specific, but multispecies approaches may be needed to formulate 
effective management strategies (Nally et al. 2002, Fischer et al. 2004). By overlaying multiple connectivity maps of several focal species, our single-species approach can be developed into a multispecies approach to identify the pathways essential for several species.

Before applying our multistep framework, two main assumptions must be critically scrutinized, and eventually, an adaptation of the framework is necessary. The first assumption is that the data collected reflects directional movement behavior. This assumption is unlikely to hold true if, during the observed time period, the investigated species has temporally distinct behavioral phases such as central place foraging, exploratory movements, or social behavior (Beier et al. 2008). If this is the case, behavioral patterns need to be distinguished and only the habitat effectively used for moving should be considered for the habitat selection analysis. Several methods can be applied to identify behavioral patterns along an animal movement track; examples are Bayesian partitioning (Calenge et al. 2009) or Brownian bridge movement models (Horne et al. 2007, Kranstauber et al. 2012). The latter additionally allows the modeling of path uncertainty related to large time intervals and high precision errors of locations. The second assumption of our analytical framework is that habitat selection is inversely related to movement resistance. Depending on the biology of the study species, low habitat selection can reflect either high physical resistance to movement or low physical resistance to movement, i.e., a habitat allowing fast movement (Stevens et al. 2006, Prevedello et al. 2010). If a positive relationship between habitat selection and movement resistance is likely, such a relationship must be considered when building alternative resistance sets.

Reduced gene flow as a result of habitat fragmentation can lead, in the longer term, to loss of genetic diversity and thus to decreased fitness, reduced adaptability to environmental changes, or even extinction of the populations which have survived (Reed 2004). Species that have survived urban development in situ might get genetically isolated through strong fragmentation of their habitat, while species that newly colonize urban areas can be restricted through barriers from spreading and colonizing further sites within a city. A reduction in genetic diversity has been detected for several vertebrates in urban areas and was explained by isolation by fragmentation (lizards and birds; Delaney et al. 2010) or by multiple independent colonization events and consequent founder effects combined with limited gene flow due to fragmentation (foxes and birds; Wandeler et al. 2003, Evans et al. 2009). In contrast, no genetic isolation was found for carabid beetles (Desender et al. 2005) and butterflies (Wood and Pullin 2002, Takami et al. 2004) in urban habitats. Although genetic structure is generally the result of processes at large temporal and spatial scales (Spear et al. 2010), the effects of movement barriers and pinch points for home range movements may be reflected in a population's genetic structure. Comparing genetic connectivity and movement-based connectivity could thus help to improve our understanding of the relationship between small-scale movements and gene flow.

Our modeling approach enables the modeling and evaluation of effects of future landscape changes on habitat connectivity. This is of special interest for predicting the development of an urban environment either under pressure of ongoing climate change (e.g., with the formation of urban heat islands) or undergoing massive transformations in its built-up area. Currently, different processes in the development of cities can be observed, such as rapid expansion of urban and suburban areas, urban densification, and sprawl (Ramalho and Hobbs 2011). These different processes have diverging effects on the urban ecosystem, but all increase habitat fragmentation. The ability to model current flow and future habitat connectivity can enable the development of sustainable strategies in urban planning and the implementation of conservation measures that take into account habitat connectivity, a fundamental requirement for maintaining and enhancing urban biodiversity.

ACKNOWLEDGMENTS

We thank K. von Wattenwyl, SWILD, for testing and reassembling the GPS transmitter units, S. Jafar-Zadeh for helping with nightly field work, and T. Sattler, WSL, for support with the data analysis. S. Hose, B. Tschander, and K. Hindenlang (City Zurich's office "Gruen Stadt Zurich" [GSZ], Fachstelle Naturschutz), and S. Steinemann and the team from the Hedgehog Centre Zurich (IZZ.ch) kindly supported the development of this study. We thank S. Dingwall for the English corrections. P. Beier and anonymous reviewers substantially helped to improve a previous version of the manuscript. The license for radio-tracking the hedgehogs was obtained from the Veterinary Office of Canton Zurich (Number $73 / 2009$ 04.05.2009). GIS layers were provided by the Swiss Federal Office of Topography (Vector25 Swisstopo, 2011, DV03594) or taken from GSZ (Biotoptypenkartierung, 2010). This study was part of the project ENHANCE (Enhancing ecosystem connectivity through intervention: A benefit for nature and society?), financed by CCES (Competence Centre Environment and Sustainability) of the ETH Zurich. M. K. Obrist and F. Bontadina shared senior authorship.

\section{Literature Cited}

Adriaensen, F., J. P. Chardon, G. De Blust, E. Swinnen, S. Villalba, H. Gulinck, and E. Matthysen. 2003. The application of 'least-cost' modelling as a functional landscape model. Landscape and Urban Planning 64:233-247.

Aebischer, N. J., P. A. Robertson, and R. E. Kenward. 1993. Compositional analysis of habitat use from animal radiotracking data. Ecology 74:1313-1325.

Beier, P., D. R. Majka, and W. D. Spencer. 2008. Forks in the road: Choices in procedures for designing wildland linkages. Conservation Biology 22:836-851.

Beyer, H. L., D. T. Haydon, J. M. Morales, J. L. Frair, M. Hebblewhite, M. Mitchell, and J. Matthiopoulos. 2010. The interpretation of habitat preference metrics under useavailability designs. Philosophical Transactions of the Royal Society B 365:2245-2254.

Biotoptypenkartierung der Stadt Zurich. 2010. NaturschutzInventar und Kartierungen. Gruen Stadt Zurich (GSZ), Zurich, Switzerland. https://www.stadt-zuerich.ch/ted/de/ index/gsz/planung_u_bau/inventare_und_grundlagen/ naturschutz-inventar_und_kartierungen.secure.html 
Bontadina, F. 1991. Strassenüberquerungen von Igeln (Erinaceus europaeus). University of Zurich, Zurich, Switzerland.

Bontadina, F., S. Gloor, and T. Hotz. 1994. Igel in der Stadt. Zürich: Wildbiologie 6(21):14.

Boyce, M. S., P. R. Vernier, S. E. Nielsen, and F. K. A. Schmiegelow. 2002. Evaluating resource selection functions. Ecological Modelling 157:281-300.

Burnham, K. P., and D. R. Anderson. 2002. Model selection and multimodel inference: a practical information-theoretic approach. Second edition. Springer, New York, New York, USA.

Calabrese, J. M., and W. F. Fagan. 2004. A comparisonshopper's guide to connectivity metrics. Frontiers in Ecology and the Environment 2:529-536.

Calenge, C. 2006. The package "adehabitat" for the R software: A tool for the analysis of space and habitat use by animals. Ecological Modelling 197:516-519.

Calenge, C., S. Dray, and M. Royer-Carenzi. 2009. The concept of animals' trajectories from a data analysis perspective. Ecological Informatics 4:34-41.

Calenge, C., and A. B. Dufour. 2006. Eigenanalysis of selection ratios from animal radio-tracking data. Ecology 87:23492355.

Carey, V. J. 2004. yags: yet another GEE solver. Version 4.02.1. R Foundation for Statistical Computing, Vienna, Austria. http://www.biostat.harvard.edu/ carey

Craiu, R. V., T. Duchesne, and D. Fortin. 2008. Inference methods for the conditional logistic regression model with longitudinal data. Biometrical Journal 50:97-109.

Cushman, S. A., K. S. McKelvey, J. Hayden, and M. K. Schwartz. 2006. Gene flow in complex landscapes: Testing multiple hypotheses with causal modeling. American Naturalist 168:486-499.

Delaney, K. S., S. P. D. Riley, and R. N. Fisher. 2010. A rapid, strong, and convergent genetic response to urban habitat fragmentation in four divergent and widespread vertebrates. PLOS ONE 5(9):e12767.

Desender, K., E. Small, E. Gaublomme, and P. Verdyck. 2005. Rural-urban gradients and the population genetic structure of woodland ground beetles. Conservation Genetics 6:51-62.

Doerr, V. A. J., T. Barrett, and E. D. Doerr. 2011. Connectivity, dispersal behaviour and conservation under climate change: a response to Hodgson et al. Journal of Applied Ecology 48:143-147.

Doncaster, C. P., C. Rondinini, and P. C. D. Johnson. 2001 Field test for environmental correlates of dispersal in hedgehogs Erinaceus europaeus. Journal of Animal Ecology 70:33-46.

Dowding, C. V., S. Harris, S. Poulton, and P. J. Baker. 2010. Nocturnal ranging behaviour of urban hedgehogs, Erinaceus europaeus, in relation to risk and reward. Animal Behaviour 80:13-21.

ESRI. 2009. ArcMap version 9.3.1. ESRI, Redlands, California, USA

Evans, K. L., et al. 2009. Independent colonization of multiple urban centres by a formerly forest specialist bird species. Proceedings of the Royal Society B 276:2403-2410.

Fieberg, J., J. Matthiopoulos, M. Hebblewhite, M. S. Boyce, and J. L. Frair. 2010. Correlation and studies of habitat selection: problem, red herring or opportunity? Philosophical Transactions of the Royal Society B 365:2233-2244.

Fieberg, J., R. H. Rieger, M. C. Zicus, and J. S. Schildcrout. 2009. Regression modelling of correlated data in ecology: subject-specific and population averaged response patterns. Journal of Applied Ecology 46:1018-1025.

Fischer, J., and D. B. Lindenmayer. 2007. Landscape modification and habitat fragmentation: a synthesis. Global Ecology and Biogeography 16:265-280.

Fischer, J., D. B. Lindenmayer, and A. Cowling. 2004. The challenge of managing multiple species at multiple scales: reptiles in an Australian grazing landscape. Journal of Applied Ecology 41:32-44.
Goddard, M. A., A. J. Dougill, and T. G. Benton. 2010. Scaling up from gardens: biodiversity conservation in urban environments. Trends in Ecology and Evolution 25:90-98.

Grimm, N. B., S. H. Faeth, N. E. Golubiewski, C. L. Redman, J. G. Wu, X. M. Bai, and J. M. Briggs. 2008. Global change and the ecology of cities. Science 319:756-760.

Halekoh, U., S. Hojsgaard, and J. Yan. 2006. The R package geepack for generalized estimating equations. Journal of Statistical Software 15:1-11.

Hardin, J. W., and J. M. Hilbe. 2003. Generalized estimating equations. Chapman and Hall, Boca Raton, Florida, USA.

Hennig, E. I., and J. Ghazoul. 2011. Plant-pollinator interactions within the urban environment. Perspectives in Plant Ecology, Evolution and Systematics 13:137-150.

Hodgson, J. A., C. D. Thomas, B. A. Wintle, and A. Moilanen. 2009. Climate change, connectivity and conservation decision making: back to basics. Journal of Applied Ecology 46:964969.

Horne, J. S., E. O. Garton, S. M. Krone, and J. S. Lewis. 2007. Analyzing animal movements using Brownian bridges. Ecology 88:2354-2363.

Koen, E. L., C. J. Garroway, P. J. Wilson, and J. Bowman. 2010. The effect of map boundary on estimates of landscape resistance to animal movement. PLoS ONE 5:8.

Koper, N., and M. Manseau. 2009. Generalized estimating equations and generalized linear mixed-effects models for modelling resource selection. Journal of Applied Ecology 46:590-599.

Kranstauber, B., R. Kays, S. D. LaPoint, M. Wikelski, and K. Safi. 2012. A dynamic Brownian bridge movement model to estimate utilization distributions for heterogeneous animal movement. Journal of Animal Ecology 81:738-746.

Laita, A., J. S. Kotiaho, and M. Monkkonen. 2011. Graphtheoretic connectivity measures: what do they tell us about connectivity? Landscape Ecology 26:951-967.

Liang, K. Y., and S. L. Zeger. 1986. Longitudinal data analysis using generalized linear models. Biometrika 73:13-22.

Manly, B. F. J., L. L. McDonald, D. L. Thomas, T. L. McDonald, and W. P. Erickson. 2002. Resource selection by animals. Statistical design and analysis for field studies. Second edition. Kluwer Academic Publisher, London, UK.

McRae, B. H. 2006. Isolation by resistance. Evolution 60:15511561.

McRae, B. H., B. G. Dickson, T. H. Keitt, and V. B. Shah. 2008. Using circuit theory to model connectivity in ecology, evolution, and conservation. Ecology 89:2712-2724.

Morris, P. A., and N. J. Reeve. 2008. Hedgehog Erinaceus europaeus. Pages 241-248 in S. Harris and D. W. Yalden, editors. Mammals of the British Isles: handbook. Mammal Society, Southampton, UK.

Nally, R. M., A. F. Bennett, G. W. Brown, L. F. Lumsden, A. Yen, S. Hinkley, P. Lillywhite, and D. Ward. 2002. How well do ecosystem-based planning units represent different components of biodiversity? Ecological Applications 12:900-912.

Pan, W. 2001. Akaike's information criterion in generalized estimating equations. Biometrics 57:120-125.

Poor, E. E., C. Loucks, A. Jakes, and D. L. Urban. 2012. Comparing habitat suitability and connectivity modeling methods for conserving pronghorn migrations. PLoS ONE 7:e49390.

Prevedello, J. A., G. Forero-Medina, and M. V. Vieira. 2010. Movement behaviour within and beyond perceptual ranges in three small mammals: effects of matrix type and body mass. Journal of Animal Ecology 79:1315-1323.

R Development Core Team. 2011. R: a language and environment for statistical computing. Reference index version 2.13.0. R Foundation for Statistical Computing, Vienna, Austria.

Ramalho, C. E., and R. J. Hobbs. 2011. Time for a change: dynamic urban ecology. Trends in Ecology and Evolution 27:179-188. 
Rayfield, B., M. J. Fortin, and A. Fall. 2011. Connectivity for conservation: a framework to classify network measures. Ecology 92:847-858.

Reed, D. H. 2004. Extinction risk in fragmented habitats. Animal Conservation 7:181-191.

Reeve, N. J. 1994. Hedgehogs. First edition. Poyser, London, UK.

Riber, A. B. 2006. Habitat use and behaviour of European hedgehog Erinaceus europaeus in a Danish rural area. Acta Theriologica 51:363-371.

Richard, Y., and D. P. Armstrong. 2010. Cost distance modelling of landscape connectivity and gap-crossing ability using radio-tracking data. Journal of Applied Ecology 47:603-610.

Rondinini, C., and C. P. Doncaster. 2002. Roads as barriers to movement for hedgehogs. Functional Ecology 16:504-509.

Sattler, T., P. Duelli, M. K. Obrist, R. Arlettaz, and M. Moretti. 2010. Response of arthropod species richness and functional groups to urban habitat structure and management. Landscape Ecology 25:941-954.

Saura, S., and L. Pascual-Hortal. 2007. A new habitat availability index to integrate connectivity in landscape conservation planning: Comparison with existing indices and application to a case study. Landscape and Urban Planning 83:91-103.

Savicky, P. 2009. pspearman: Spearman's rank correlation test. Version 0.2-5. R Foundation for Statistical Computing, Vienna, Austria. http://cran.r-project.org/web/packages/ pspearman/index.html

Sawyer, S. C., C. W. Epps, and J. S. Brashares. 2011. Placing linkages among fragmented habitats: do least-cost models reflect how animals use landscapes? Journal of Applied Ecology 48:668-678.

Shafer, A. B. A., J. M. Northrup, K. S. White, M. S. Boyce, S. D. Côté, and D. W. Coltman. 2012. Habitat selection predicts genetic relatedness in an alpine ungulate. Ecology 93:1317-1329.

Shirk, A. J., D. O. Wallin, S. A. Cushman, C. G. Rice, and K. I. Warheit. 2010. Inferring landscape effects on gene flow: a new model selection framework. Molecular Ecology 19:36033619.

Smith, R. M., P. H. Warren, K. Thompson, and K. J. Gaston. 2006. Urban domestic gardens (VI): environmental correlates of invertebrate species richness. Biodiversity and Conservation 15:2415-2438.

Spear, S. F., N. Balkenhol, M. J. Fortin, B. H. McRae, and K. Scribner. 2010. Use of resistance surfaces for landscape genetic studies: considerations for parameterization and analysis. Molecular Ecology 19:3576-3591.

Stevens, V. M., E. Leboulenge, R. A. Wesselingh, and M. Baguette. 2006. Quantifying functional connectivity: experi- mental assessment of boundary permeability for the natterjack toad (Bufo calamita). Oecologia 150:161-171.

Swisstopo. 2011. Vector25. Das digitale Landschaftsmodell der Schweiz. Bundesamt für Landestopographie, Wabern, Switzerland. http://www.swisstopo.admin.ch/internet/swisstopo/ de/home/products/landscape/vector $25 . h$ tml

Takami, Y., C. Koshio, M. Ishii, H. Fujii, T. Hidaka, and I. Shimizu. 2004. Genetic diversity and structure of urban populations of Pieris butterflies assessed using amplified fragment length polymorphism. Molecular Ecology 13:245258

Taylor, P. D., L. Fahrig, K. Henein, and G. Merriam. 1993. Connectivity is a vital element of landscape structure. Oikos 68:571-573.

Taylor, P. D., L. Fahrig, and K. A. With. 2006. Landscape connectivity: a return to the basics. Pages $29-43$ in K. R. Crooks and M. Sanjayan, editors. Connectivity conservation. Cambridge University Press, New York, New York, USA.

Trainor, A. M., J. R. Walters, W. F. Morris, J. Sexton, and A. Moody. 2013. Empirical estimation of dispersal resistance surfaces: a case study with red-cockaded woodpeckers. Landscape Ecology 28:755-767.

United Nations. 2012. World urbanization prospects: The 2011 revision. United Nations, New York, New York, USA.

Urban, D., and T. Keitt. 2001. Landscape connectivity: a graph-theoretic perspective. Ecology 82:1205-1218.

Urban, D. L., E. S. Minor, E. A. Treml, and R. S. Schick. 2009. Graph models of habitat mosaics. Ecology Letters 12:260 273.

Wandeler, P., S. M. Funk, C. R. Largiader, S. Gloor, and U. Breitenmoser. 2003. The city-fox phenomenon: genetic consequences of a recent colonization of urban habitat. Molecular Ecology 12:647-656.

White, G. C., and R. A. Garrott. 1990. Analysis of wildlife radio-tracking data. Academic Press, San Diego, California, USA.

Wood, B. C., and A. S. Pullin. 2002. Persistence of species in a fragmented urban landscape: the importance of dispersal ability and habitat availability for grassland butterflies. Biodiversity and Conservation 11:1451-1468.

$\mathrm{Xu}$, Q.-S., and Y.-Z. Liang. 2001. Monte Carlo cross validation. Chemometrics and Intelligent Laboratory Systems 56:1-11.

Young, R. P., J. Davison, I. D. Trewby, G. J. Wilson, R. J. Delahay, and C. P. Doncaster. 2006. Abundance of hedgehogs (Erinaceus europaeus) in relation to the density and distribution of badgers (Meles meles). Journal of Zoology 269:349-356.

Zingg, R. 1994. Aktivität sowie Habitat- und Raumnutzung von Igeln (Erinaceus eruopaeus) in einem ländlichen Siedlungsgebiet. Universität Zürich, Zürich, Switzerland.

\section{Supplemental Material}

Appendix A

Habitat classes used for habitat selection and connectivity analyses (Ecological Archives A024-193-A1).

Appendix B

Calculation of the average habitat selection axis (Ecological Archives A024-193-A2).

Appendix C

Details about the GPS tracking data of urban hedgehogs (Ecological Archives A024-193-A3).

\section{Appendix D}

Pairwise comparison between the five alternative connectivity models (Ecological Archives A024-193-A4). 